\title{
Diagnostic Accuracy of Cardiac Magnetic Resonance Versus Fractional Flow Reserve: A Systematic Review and Meta-Analysis
}

\author{
Waqas Ullaha, g, Sohaib Roomi ${ }^{\mathrm{a}}$, Hafez M. Abdullah ${ }^{\mathrm{b}}$, Maryam Mukhtarc, \\ Zain Alia ${ }^{a}$ Ping Ye ${ }^{b}$, d, Donald C. Haas ${ }^{e}$, Vincent M. Figueredo ${ }^{f}$
}

\begin{abstract}
Background: Fractional flow reserve (FFR) is considered the gold standard for diagnosis of coronary artery disease (CAD). Stress Cardiac magnetic resonance (SCMR) has been recently gaining traction as a non-invasive alternative to FFR.
\end{abstract}

Methods: Studies comparing the diagnostic accuracy of SCMR versus FFR were identified and analyzed using Review Manager (RevMan) 5.3 and Stata software.

Results: A total of 28 studies, comprising 2,387 patients, were included. The pooled sensitivity and specificity for SCMR were $86 \%$ and $86 \%$ at the patient level, and $82 \%$ and $88 \%$ at the vessel level, respectively. When the patient-level data were stratified based on the FFR thresholds, higher sensitivity and specificity (both 90\%) were noted with the higher cutoff (0.75) and lower cutoff (0.8), respectively. At the vessel level, sensitivity and specificity at the lower FFR threshold were significantly higher at $88 \%$ and $89 \%$, compared to the corresponding values for higher cutoff at 0.75 . Similarly, meta-regression analysis of SCMR at higher (3T) resolution showed a higher sensitivity of $87 \%$ at the patient level and higher specificity of $90 \%$ at the vessel level. The highest sensitivity and specificity of SCMR $(92 \%$ and $94 \%$, respectively) were noted in studies with CAD prevalence greater than $60 \%$.

Conclusions: SCMR has high diagnostic accuracy for CAD comparable to FFR at a spatial resolution of $3 \mathrm{~T}$ and an FFR cut-off of 0.80 . An increase in CAD prevalence further improved the specificity of SCMR.

Manuscript submitted February 5, 2020, accepted February 26, 2020

anternal Medicine, Abington Hospital-Jefferson Health, Abington, PA, USA

b Internal Medicine, University of South Dakota, Sioux Falls, SD, USA

'Internal Medicine, Fauji Foundation Hospital, Rawalpindi, Pakistan

dAvera Research Institute, Avera Health, Sioux Falls, SD, USA

'Abington Hospital-Jefferson Health, Abington, PA, USA

fSt. Mary Medical Center, Langhorne, PA, USA

gCorresponding Author: Waqas Ullah, Internal Medicine, Abington Hospital-

Jefferson Health, Abington, PA 19001, USA.

Email: waqasullah.dr@gmail.com

doi: https://doi.org/10.14740/cr1028
Keywords: Cardiac magnetic resonance; Fractional flow reserve; Coronary stenosis

\section{Introduction}

The interpretation of invasive coronary angiography (ICA) based on anatomic reference standard has been the sole diagnostic modality for coronary artery disease (CAD) for a long time [1]. However, fractional flow reserve (FFR) and stress cardiac magnetic resonance (SCMR) are now coming into more frequent use. Recent studies have demonstrated the discordance between FFR and ICA, especially at the extremes of the angiographic spectrum (stenosis less than $30 \%$ or greater than 90\%) [1]. Thus, an assumption of functional relevance based merely on the angiographic appearance of stenosis in the absence of FFR can be misleading. FFR appears to be more precise for the depiction of lesion-specific ischemia but at the cost of radiation exposure and an invasive procedure. SCMR perfusion scans have been suggested as an alternative to guide clinical decision-making, particularly in cases of CAD with stenosis of $50-75 \%$ [2]. Our study adds to the existing literature by pooling all available data to determine the diagnostic accuracy of SCMR for diagnosing CAD severity when compared to the gold-standard FFR.

\section{Materials and Methods}

\section{Search strategy}

A literature search for relevant articles was performed using PubMed, Ovid, Embase, clinicaltrials.gov, and Cochrane databases. There was no language or time restriction. The search strategies included various combinations of text-words and medical subject headings (MeSH) to generate two subsets of citations: one for magnetic resonance imaging using the terms such as "MRI", "magnetic resonance imaging", "magnetic resonance perfusion", "cardiac MRI", "SCMRI", "MR perfusion imaging"; the other for fractional flow reserve using terms such as "FFR", "fractional flow reserve", "flow reserve", 
"coronary flow reserve", and "coronary pressure reserve". The terms from the two subsets were combined in 1:1 combination using Boolean operators; and finally, results from all the possible combinations were downloaded into an EndNote library. Based on our research question, articles from the reference lists relevant to the clinical question were screened by an independent author.

\section{Selection criteria and study collection}

All observational (retrospective/prospective) studies and randomized control trials (RCT) on SCMR and FFR till September 30, 2019, were evaluated. Articles comparing the diagnostic accuracy of SCMR perfusion scan with FFR were included in the study. Papers with insufficient data, review articles, case reports, editorials, and conference papers were excluded.

\section{Data extractions}

Three authors independently screened titles and abstracts of all articles for relevance. A fourth author selected articles that met inclusion criteria. The full texts of articles that were potentially relevant to the study were screened by all the four authors to confirm eligibility. Baseline characteristics of the included population were reviewed, and data were collected for quality assessment to ensure study cohorts were statistically comparable. Risk factors for CAD, such as hypertension (HTN), hyperlipidemia (HLD), smoking, diabetes mellitus (DM), prior myocardial infarction (MI) and prior CAD were compared in each group of the included study to ensure comparability of the included population. Data were extracted into a combined Excel sheet. Disagreements were resolved by mutual consensus and after group discussion. The primary outcome was to determine the diagnostic accuracy of SCMR versus FFR for CAD severity.

\section{Data and quality analysis}

Raw data regarding the true and false positives and true and false negatives of each included study were obtained; and the combined measures of test accuracy (i.e., sensitivity, specificity, and diagnostic odds ratio (DOR)) for SCMR perfusion were calculated, keeping FFR as a standard. A bivariate model was obtained from data fitting. The parameters from the bivariate model were transformed into hierarchical summary receiver operating characteristic (HSROC) estimates. The area under the summary HSROC curve (AUC) was calculated to reveal the probability of accurately ranked diagnostic test values for a random pair of subjects (one with disease and one without disease). Chi-squared $\left(\chi^{2}\right)$ tests were conducted to evaluate the heterogeneity of sensitivities and specificities among all studies. Subgroup analysis based on the resolution of SCMR and FFR cutoff points was performed to explore the potential heterogeneity factors using subgroup and meta-regression analysis. The statistical analysis was performed using the diagnostic

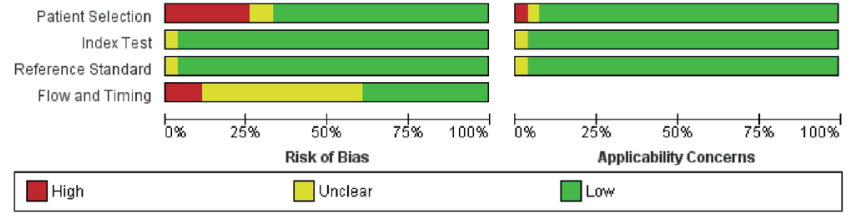

Figure 1. Overall risk of bias on the QUADAS-2 tool of the included studies in our study. QUADAS-2: the Quality Assessment of Diagnostic Accuracy Studies.

accuracy statistical model on Stata and R mad package.

\section{Quality of included studies}

Quality of the included studies was assessed for potential bias and applicability concerns using the Quality Assessment of Diagnostic Accuracy Studies (QUADAS-2) standard questionnaire. The overall risk of bias and applicability concerns based on patient selection, index and reference standard, and the time duration between these tests were of low concern. Approximately $40 \%$ of studies either did not mention the duration or had more than 4 weeks difference between the aforementioned diagnostic tests. Only one study (Schuchlenz et al) had insufficient bias assessment data posing a 3\% unknown bias risk to the index and reference standard tests [3] (Fig. 1). Twenty-five percent of the studies did not specify the baseline characteristics of its subjects and were stratified under the high-risk selection bias group (Fig. 2).

\section{Results}

\section{Search results and study characteristics}

The initial search revealed 3,921 articles. After the removal of irrelevant and duplicate items, 238 articles were deemed relevant for full-text review. We further excluded 210 articles based on our selection criteria; and 28 studies were included for final analysis [3-30]. The preferred reporting items for systematic review and meta-analysis (PRISMA) flow diagram are shown in Figure 3.

A total of 2,387 patients and 3,936 coronary vessels were included [3-30]. Mean age was 63 years. Seventeen studies used 0.8 , and 11 articles used 0.75 as FFR cutoff. Studies using both $1.5 \mathrm{~T}$ (18) and 3.0T (10) SCMR spatial resolution were included. Semi-quantitative data analysis was employed in 10 studies, quantitative in nine studies, while the method of data analysis was not specified in the remainder. A time interval of 4 weeks or less was present between SCMR and FFR in 16 of the included studies, more than 4 weeks in three studies, and not specified in eight studies. Subjects included in all the available studies had a variable prevalence of the baseline characteristics and comorbidities, including prior MI, CAD, DM, HTN, multivessel disease, and smoking. The detailed characteristics of the included studies are summarized (Supplementary Material 1, www.cardiologyres.org). 


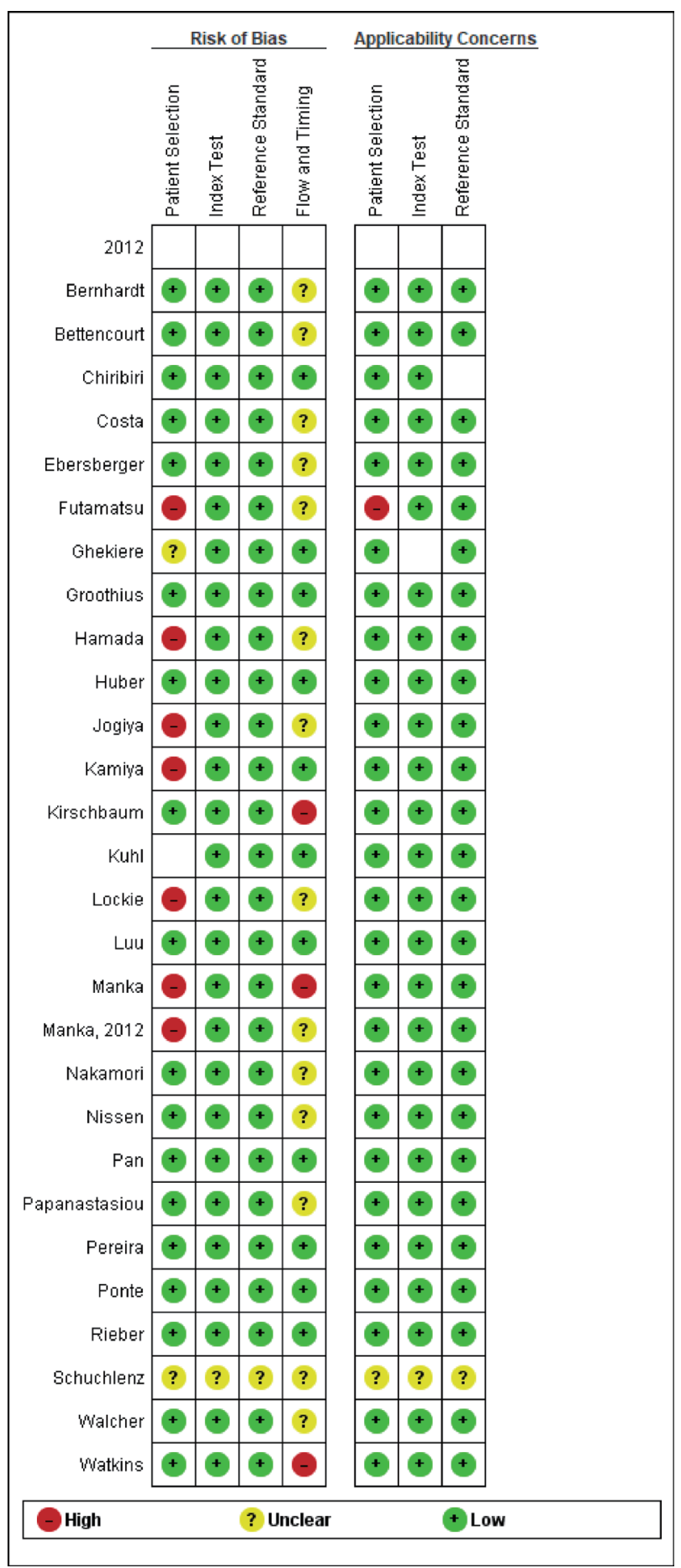

Figure 2. Risk of bias on the QUADAS-2 tool of the individual studies included in our meta-analysis. QUADAS-2: the Quality Assessment of Diagnostic Accuracy Studies.

\section{Patient-level pooled diagnostic accuracy}

At the patient level, pooled sensitivity and specificity for perfusion SCMR were 0.86 (95\% confidence interval (CI), 0.79 - 0.91) and 0.86 (95\% CI, 0.82 - 0.90), respectively (Fig. 4a,

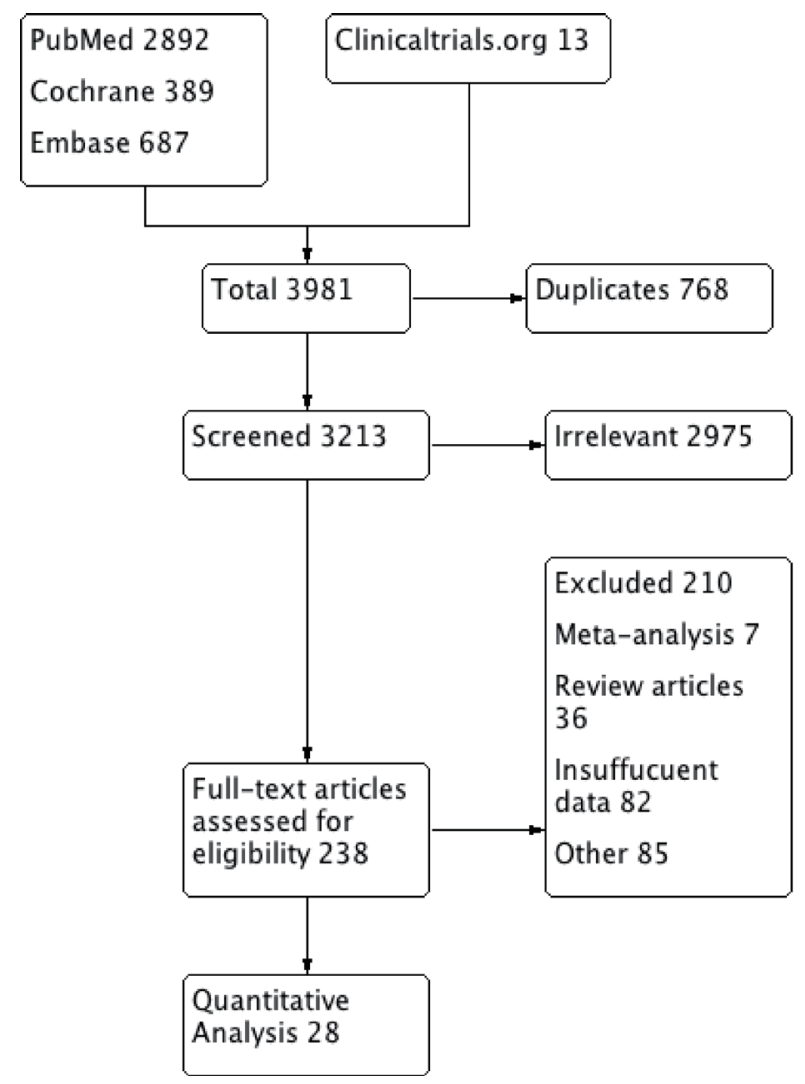

Figure 3. PRISMA flow diagram showing the selection of studies from all databases. PRISMA: preferred reporting items for systematic review and meta-analysis.

b). The DOR on a per-patient basis was 48.5 (95\% CI, 23.52 100.41). The absolute AUC was 0.91, while partial AUC was 0.78 (Fig. 5). The test for equality of sensitivities showed moderate to severe heterogeneity $\mathrm{I}^{2}=88 \%\left(\chi^{2}=137.17, \mathrm{df}=16, \mathrm{P}\right.$ value $\leq 2 \times 10^{-16}$ ), while moderate heterogeneity was observed among the studies selected for equality of pooled specificities $\mathrm{I}^{2}=66 \%\left(\chi^{2}=50.59, \mathrm{df}=16, \mathrm{P}\right.$ value $\left.=1.85 \times 10^{-5}\right)$.

\section{Coronary-territory pooled diagnostic accuracy}

At vessel level, SCMR showed an overall sensitivity of 0.82 ( $95 \%$ CI, $0.76-0.88$ ), and specificity of 0.88 (95\% CI, 0.84 0.94) compared to FFR (Fig. 6a, b). The absolute AUC, partial AUC and DOR were 0.91, 0.85 and 37.95 (95\% CI, 22.60 63.73), respectively (Fig. 7). The test for equality of diagnostic accuracy showed moderate to severe heterogeneity among the studies selected for sensitivity $\left(\mathrm{I}^{2}=89 \%, \chi^{2}=204.5, \mathrm{df}=21, \mathrm{P}\right.$ $\left.\leq 2 \times 10^{-16}\right)$, and specificity $\left(\mathrm{I}^{2}=85 \%, \chi^{2}=142.9, \mathrm{df}=21, \mathrm{P} \leq\right.$ $\left.2 \times 10^{-16}\right)$

\section{Subgroup analysis based on FFR threshold}

Pooled diagnostic accuracy was stratified based on the two reported FFR thresholds. At the vessel level, sensitivity and 


a Study name
Bernhardt 2012
Bettencourt 2013
Chiribiri 2013
Ebersberger 2013
Futamatsu 2007
Groothius 2013
Hamada 2017
Jogiya 2012
Manka 2012
Manka 2015
Nakamori 2017
Nissen 2018
Papanastasiou 2016
Pereira 2013
Ponte 2014
Walcher 2012
Watkins 2009
Combined

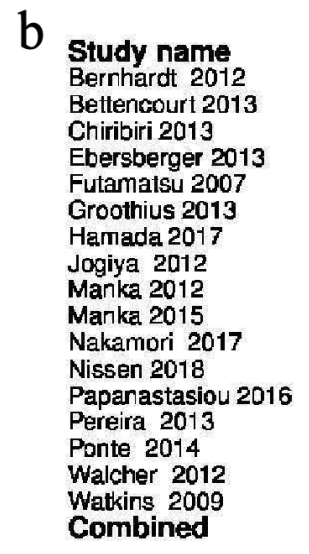

$\begin{array}{rr}\text { Sensitivity } & \text { Lower limit } \\ 0.62 & 0.41 \\ 0.89 & 0.76 \\ 0.91 & 0.8 \\ 0.85 & 0.71 \\ 0.82 & 0.52 \\ 0.85 & 0.66 \\ 0.96 & 0.92 \\ 0.91 & 0.77 \\ 0.9 & 0.81 \\ 0.85 & 0.76 \\ 0.88 & 0.78 \\ 0.41 & 0.29 \\ 0.94 & 0.72 \\ 0.81 & 0.66 \\ 0.88 & 0.74 \\ 0.88 & 0.66 \\ 0.95 & 0.88 \\ \mathbf{0 . 8 6} & \mathbf{0 . 7 9}\end{array}$
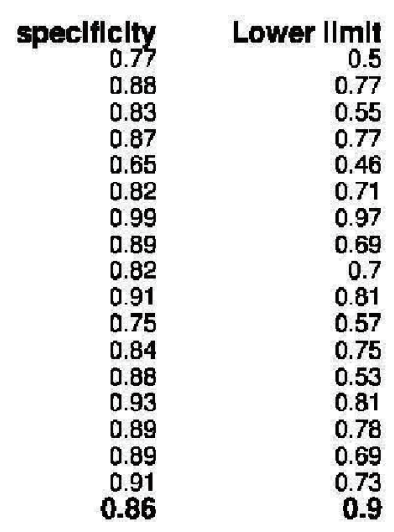
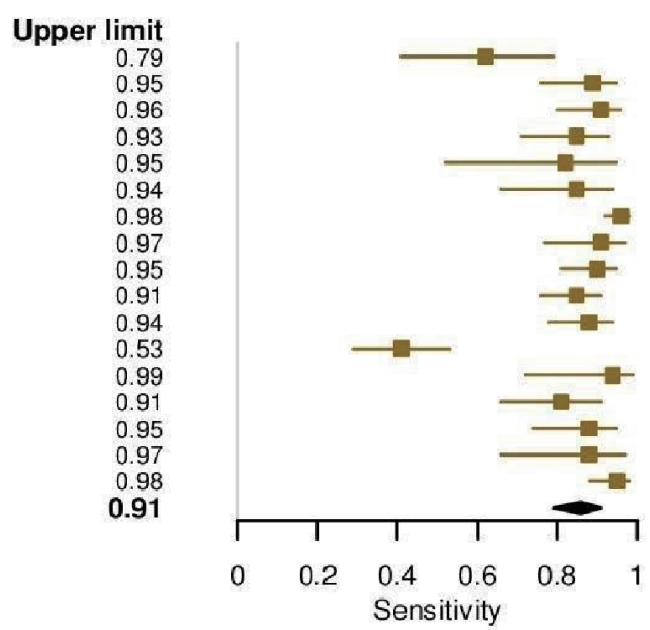
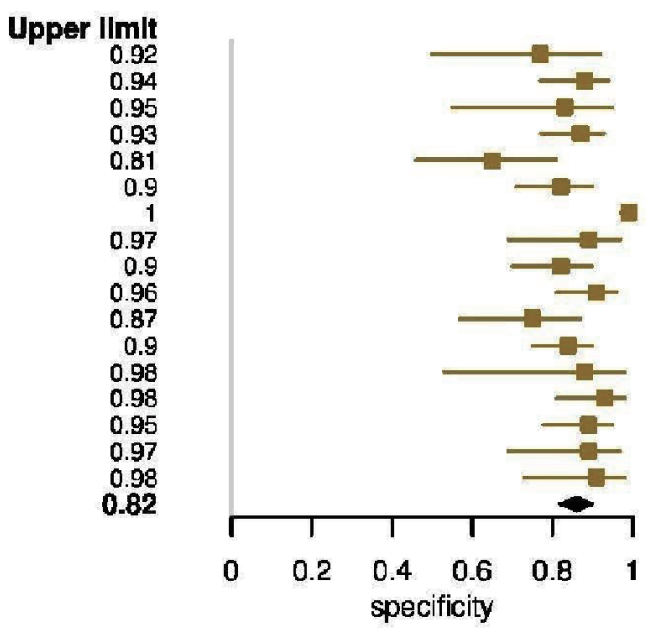

Figure 4. (a) Forest plot depicting individual and pooled sensitivity at the patient level. (b) Forest plot depicting individual and pooled specificity at the patient level.

specificity at the lower FFR threshold (0.8) were significantly higher at $0.88(95 \% \mathrm{CI}, 0.84-0.91)$ and 0.89 (95\% CI, 0.82 - 0.93), compared to $0.80(0.68-0.88)$ and $0.87(0.82-0.91)$ for FFR at the higher threshold (0.75) (Supplementary Material 2, www.cardiologyres.org). The DOR of FFR at the higher threshold was only 29.71 , almost half of the DOR for the lower (0.80) cutoff (62.37 (95\% CI, 32 - 118).

At the patient level, however, the higher cutoff exhibited a higher net sensitivity, 0.90 (95\% CI, 0.84 - 0.94) but lower specificity $0.82(95 \% \mathrm{CI}, 0.73-0.88)$ compared to the lower cutoff, where the sensitivity was 0.85 (95\% CI, $0.76-0.91)$ and specificity was 0.90 (95\% CI, $0.84-0.93)$ (Supplementary Material 3, www.cardiologyres.org). The overall DOR favored the lower FFR threshold $(52.3,95 \%$ CI, 20.81 - 131.47) vs. (43.96, 95\% CI, 18.98 - 101.84).

\section{Subgroup analysis based on SCMR resolution}

At vessel level, the specificity of SCMR at higher resolution
(0.90, $95 \%$ CI 0.866 - 0.93) was significantly greater compared to lower resolution $(0.85,95 \%$ CI, 0.79 - 0.89) (Supplementary Material 4, www.cardiologyres.org), while the sensitivity was marginally higher for the lower resolution (0.85 vs. 0.81$)$. The overall DOR was superior for higher resolution SCMR (44, 95\% CI, 20 - 95) compared to $(33,95 \%$ CI, 16 - 66) for 1.5T SCMR. At the patient level, the sensitivity and specificity were comparable at both resolutions (Supplementary Material 5, www.cardiologyres.org).

\section{Meta-regression analysis based on comorbidities}

Meta-regression analysis based on covariates such as age (higher or lower than 65 years) and CAD prevalence were performed. SCMR had high sensitivity and specificity at 0.92 and 0.94 , respectively, for studies with CAD prevalence greater than $60 \%$, compared with the sensitivity of 0.83 , and specificity of 0.84 with prevalence of CAD less than $60 \%$ (Supplementary Material 6, www.cardiologyres.org). Corre- 


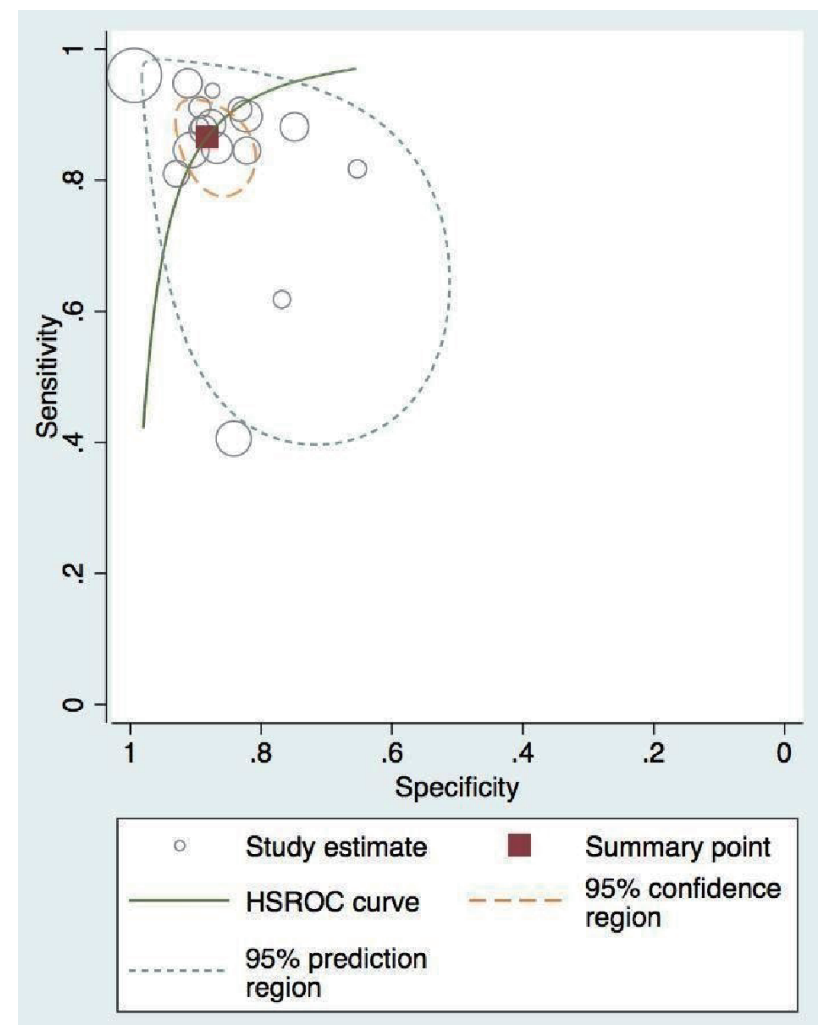

Figure 5. Patient-level pooled diagnostic accuracy of CMR associated HROC vessel. CMR: cardiac magnetic resonance; HROC: hierarchical summary receiver operating characteristic.

sponding values at the vessel level were lower (Supplementary Material 6, www.cardiologyres.org). For both younger and older populations, the SCMR sensitivity was 0.86 , but a higher specificity was noted for the younger population $(0.89$ vs. 0.81) (Table 1). A comparison of patient and vessel level data has been shown in Supplementary Material 7 (www.cardiologyres.org).

\section{Discussion}

If proven to have similar diagnostic efficacy, SCMR would be favorable to the current reference standard (FFR) due to its non-invasive nature, zero radiation exposure, and lower attenuation artifacts. Although myocardial perfusion SCMR has been possible for many years, the clinical sequence and data processing remain variable between centers. Our metaanalysis estimated the diagnostic performance of perfusion SCMR in patients with known or suspected CAD and focused on comparing diagnostic accuracies at varying spatial resolution and FFR thresholds. Our results reconcile the varying findings of previous studies and show high diagnostic accuracy of SCMR perfusion using FFR as a reference at patient level, with a sensitivity of 0.86 and specificity of 0.86 . Subgroup analysis for the FFR threshold of lower (0.8) compared to higher $(0.75)$ showed better sensitivity and specificity (about 90\%) for the former. Further analysis showed that for higher spatial resolution of SCMR (3.0T), the sensitivity and specificity were also greater at $87 \%$ and $90 \%$, respectively.

FFR is calculated as the ratio of hyperemic pressure difference across coronary lesions compared to theoretical maximum flow in healthy coronary arteries [31]. FFR is considered the gold standard and has been proven to improve event-free survival in coronary revascularization [32]. However, for all its benefits, it has yet to gain widespread acceptance due to the invasive nature of the procedure [32]. As mentioned above, SCMR perfusion imaging, which is a non-invasive procedure, would seek to allay the concerns around the risks of FFR while offering a similar diagnostic profile. Interpretation is based on coronary flow reserve (CFR), which is driven by the pressure gradient between systemic diastolic and left ventricular enddiastolic pressure at maximal vasodilation and under medication-induced stress. With vasodilator CMR (particularly using regadenoson, the most commonly used vasodilator currently), the perfusion defects are related to relative differences in blood flow between myocardium subtended by normal vs. stenotic coronary arteries [31]. The percentage of the ischemic territory serves as an indirect indicator of coronary artery stenosis, and is correlated against the findings of FFR [32].

On review of previously published meta-analyses, we noticed inconsistencies in the number of included studies. More contemporary studies did not always include all previously published data and had additional limitations due to outcome biases [2, 33-37]. Studies more stringent in their inclusion criteria, perhaps overestimated the diagnostic accuracy of SCMR owing to a positive selection bias. Danad et al (2016, four studies) reported a patient-based SCMR accuracy of greater than $90 \%$; however, this was found to be lower at $87 \%$ in a 2019 study by Yang et al, which included a much higher number of studies (19) [2,36]. Vessel-level sensitivity analysis showed a similar discordance across all included studies. A common limitation observed was a lack of stratification based on the different diagnostic thresholds for SCMR perfusion scans and FFR [34-37]. The study of Desai et al was the only one that used an FFR diagnostic threshold of 0.75 as opposed to the standard 0.80 , hampering the sensitivity of the SCMR perfusion scan by classifying a significant population of patients having FFR of $0.75-0.80$ as falsely "normal" [33]. Similarly, many included studies used $40-80 \%$ stenosis as inclusion criteria for stable $\mathrm{CAD}$, when compared to the standard criteria of $50-75 \%$ stenosis. This would falsely classify subjects as having CAD compromising the sensitivity of SCMR (Table 2, [2, 33-37]). Moreover, differences in the contrast used across the studies also undermined the results; a few authors used exogenous contrast for stress images, while others advocated the utilization of the paramagnetic properties of deoxyhemoglobin as an endogenous contrast to identify loss of T2 flair in the blood oxygen level-dependent (BOLD) SCMR [36].

We aimed to close the gap in clinical practice and obtain consensus on the preferred approach towards the diagnosis of CAD severity, by performing a comprehensive analysis of all available data to address the aforementioned limitations.

The objective was to compare the efficacy of two investigative strategies for the management of patients with suspected 

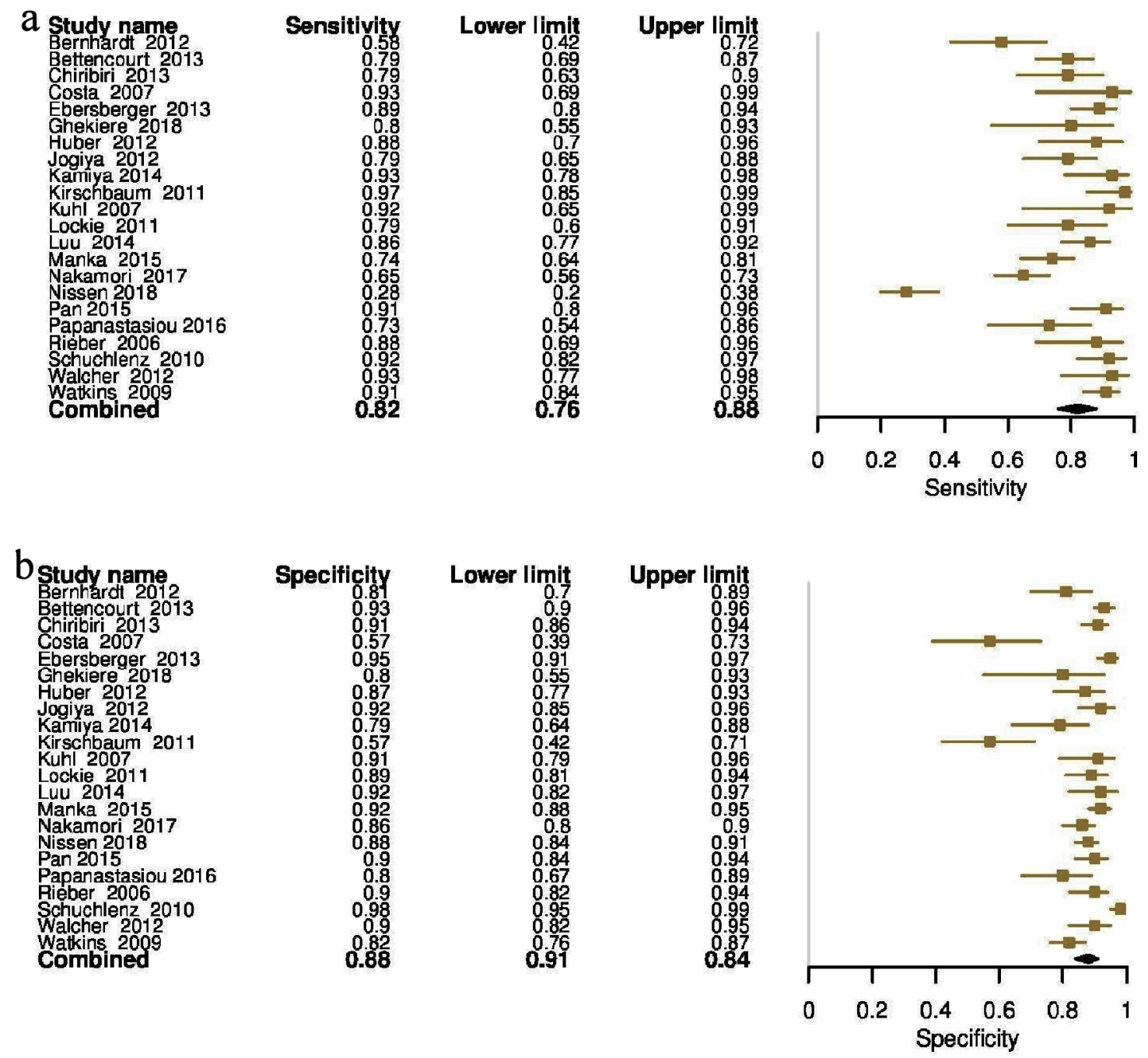

Figure 6. (a) Forest plot depicting individual and pooled sensitivity at the vessel level. (b) Forest plot depicting individual and pooled specificity at the vessel level.

CAD at the level of both higher and lower FFR thresholds and SCMR spatial resolution. Our overall results indicate that at a lower FFR threshold and higher spatial resolution, SCMR perfusion is comparable to invasive angiography supplemented by FFR measurements in guiding the management of patients with stable CAD. Our subgroup analyses showed a $4 \%$ rise in the vessel-level specificity of SCMR with an increase in its resolution from $1.5 \mathrm{~T}$ to $3.0 \mathrm{~T}$ and an $8 \%$ increase in specificity with the decreasing FFR threshold from 0.75 to 0.80 . This improves the detection ability of SCMR perfusion to identify subendocardial ischemia. It also enables the detection of stress-mediated regional wall motion abnormalities at an earlier stage [36]. Given the comparable accuracy of SCMR perfusion scan to the current invasive reference standard (FFR), SCMR should be seen as an attractive non-invasive alternative to current diagnostic approaches.

This meta-regression analysis showed that sensitivity and specificity of SCMR differed among populations with differing pre-test probability of $\mathrm{CAD}$, indicating patient selection based on presentation and risk stratification is essential. In the high prevalence group, SCMR findings were equivalent to FFR, and overall specificity was high at $94 \%$. In these selected patients, SCMR can serve as a non-invasive substitute for FFR without significant loss in diagnostic accuracy. Interestingly, meta-regression based on age cutoff of 65 years showed similar sensitivity but an $8 \%$ lower specificity in the older population. One can argue that microvascular disease as a result of advancing age, smoking, and atherosclerosis can potentially affect SCMR perfusion imaging more than the FFR, and can have a detrimental effect on diagnostic accuracy. Stress CMR perfusion defects can be due to epicardial CAD and/or microvascular CAD. FFR on the other hand can be falsely in the "normal" range because of severe microvascular CAD, even in the presence of epicardial CAD. Thus, stress CMR is arguably more sensitive because unlike FFR, it "accounts for" the possibility of microvascular CAD, and the diagnostic accuracy comparisons may actually be affected because of a flawed reference standard (FFR). 


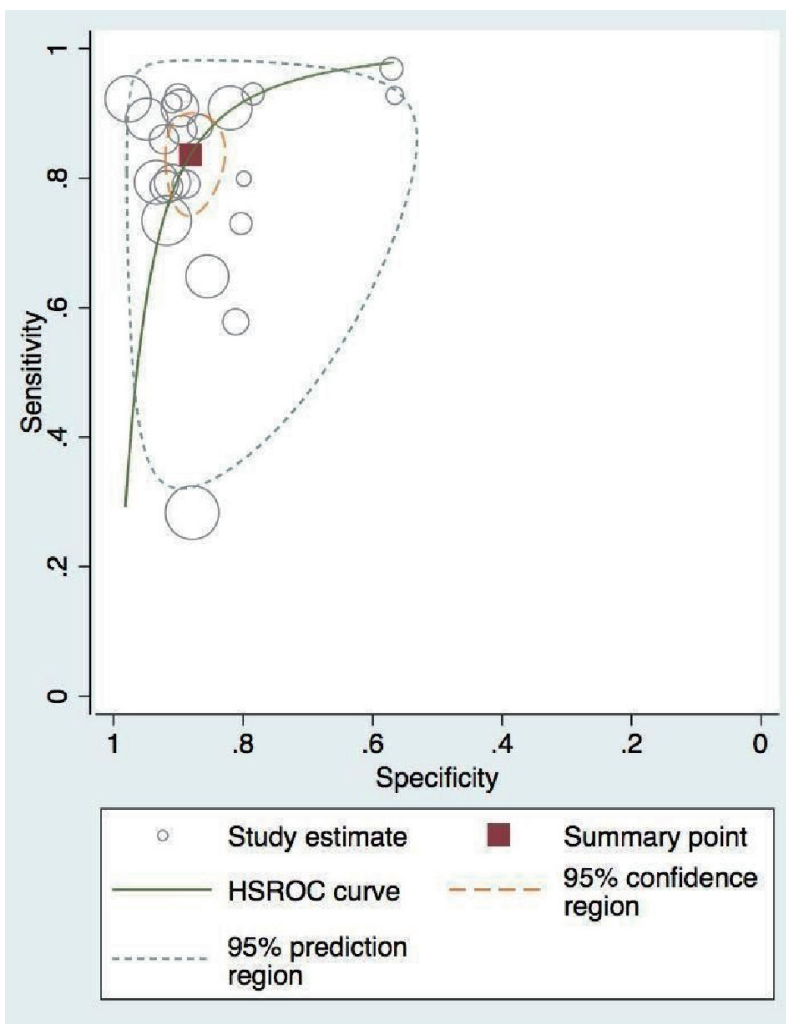

Figure 7. Vessel-level pooled diagnostic accuracy of CMR associated HROC. CMR: cardiac magnetic resonance; HROC: hierarchical summary receiver operating characteristic.

\section{Limitations}

A few of the included studies performed a direct comparison of SCMR perfusion scan with FFR, an observation that may underscore the restricted availability of SCMR to advanced centers. Paradoxically, a lower rate of diagnostic accuracy is expected when SCMR becomes more widely available, leading to possible imprudent use.

We believe that the conceptual differences between these tests are irrefutable. FFR assumes static endothelial function on either side of the coronary lesion and an intact microcirculation. Thus, findings are reliable in large coronary arteries but are often underappreciated in microcirculation. In contrast, SCMR assesses the coronary vasculature in its entirety. FFR identifies the hemodynamic significance and functional relevance of coronary lesions, but gives no direct indication of the size of territory at risk of ischemia or its viability. SCMR, on the other hand, is a surrogate marker of coronary stenosis, an indirect measure of the degree of functional and anatomic coronary compromise based on the identification of myocardial ischemia. This technical discrepancy poses some theoretical risk of inconsistency and unreliability between the two diagnostic modalities.

\section{Conclusions}

SCMR can be used as a confirmation test for patients with a high pretest probability of CAD and as an exclusionary test for low-risk patients due to its high specificity and sensitivity compared to FFR, respectively. A lower FFR threshold of

Table 1. Meta-Regression Analysis on the Basis of CAD Prevalence and Age of the Patients in Included Studies

\begin{tabular}{llll}
\hline Variable & Sensitivity $\mathbf{( 9 5 \%}$ CI) & Specificity (95\% CI) & DOR \\
\hline Vessel level, MI $>60 \%$ & $0.84(0.77-0.88)$ & $0.86(0.81-0.89)$ & $32(19-53)$ \\
Patient, MI $>60 \%$ & $0.92(0.88-0.94)$ & $0.94(0.92-0.96)$ & $12(10-33)$ \\
Vessel level, MI $<60 \%$ & $0.81(0.66-0.91)$ & $0.88(0.82-0.92)$ & $34(14-80)$ \\
Patient, MI $<60 \%$ & $0.82(0.74-0.89)$ & $0.84(0.81-0.87)$ & $27(15-50)$ \\
Vessel level, Age $<65$ & $0.82(0.74-0.88)$ & $0.89(0.85-0.92)$ & $34(14-80)$ \\
Patient, Age $<65$ & $0.86(0.79-0.91)$ & $0.89(0.85-0.93)$ & $58(26-126)$ \\
Vessel level, Age $>65$ & $0.86(0.71-0.94)$ & $0.81(0.72-0.88)$ & $28(12-67)$ \\
\hline
\end{tabular}

CAD: coronary artery disease; MI: myocardial infarction; 95\% Cl: 95\% confidence interval; DOR: diagnostic odds ratio.

Table 2. Characteristics, Outcomes and Limitations of Previously Reported Meta-Analyses

\begin{tabular}{lllll}
\hline \multirow{2}{*}{ Author } & Studies included & \multicolumn{2}{c}{ Sensitivity, specificity } & Limitation \\
\cline { 3 - 5 } & & Patient level & Vessel level & \\
\hline Desai et al, 2013 [33] & 12 & $89.1 \%, 84.9 \%$ & $87.7 \%, 88.6 \%$ & Used 0.75 only \\
\hline Li et al, 2014 [34] & 14 & $90 \%, 87 \%$ & $89 \%, 86 \%$ & No stratification based on thresholds \\
Jiang et al, 2016 [35] & 20 & $88 \%, 88 \%$ & $86 \%, 88 \%$ & No stratification based on thresholds \\
Danad et al, 2017 [36] & 4 & $90 \%, 94 \%$ & $91 \%, 85 \%$ & No stratification based on thresholds \\
Dai et al, 2016 [37] & 21 & $88 \%, 84 \%$ & $87 \%, 89 \%$ & No stratification based on MRI thresholds \\
Yang et al, 2019 [2] & 19 & $87 \%, 87 \%$ & $85 \%, 89 \%$ & CAD 50-75\% \\
\hline
\end{tabular}

CAD: coronary artery disease. 
0.8 and a higher SCMR resolution of 3.0T, in particular, were associated with the highest diagnostic accuracy. SCMR represents advances over FFR in the detection of CAD severity, due to its non-invasive nature, being readily available and offering a comparable sensitivity and specificity.

\section{Supplementary Material}

Suppl 1. Characteristics of Included Studies.

Suppl 2. HROC for FFR of 0.75 and 0.8 at the vessel level.

Suppl 3. HROC for FFR of 0.75 and 0.8 at the patient level.

Suppl 4. HROC for CMR at vessel level at spatial resolution of $1.5 \mathrm{~T}$ and $3 \mathrm{~T}$.

Suppl 5. HROC for CMR at patient level at spatial resolution of $1.5 \mathrm{~T}$.

Suppl 6. Meta-regression based on the prevalence of MI < $60 \%$ at the patient level, MI $>60 \%$ at the vessel level, and MI $<60 \%$ at the vessel level.

Suppl 7. Meta-regression based on the age $<65$ at the patient level, $<65$ at the patient level, and $>65$ at the vessel level.

\section{Acknowledgments}

We thank Dr. Margot Boigon for providing research opportunities and resources in the institute.

\section{Financial Disclosure}

None to declare.

\section{Conflict of Interest}

None to declare.

\section{Informed Consent}

Not applicable.

\section{Author Contributions}

Waqas Ullah: conceptualization, manuscript writing, and analysis; Sohaib Roomi: discussion; Hafez M. Abdullah: literature search; Maryam Mukhtar: data collection; Zain Ali: data mining; Ping Ye: figures creation; Donald Haas: critical review; Vincent Figueredo: supervision.

\section{Data Availability}

The authors declare that data supporting the findings of this study are available within the article.

\section{Abbreviations}

CMR: cardiac magnetic resonance; CAD: coronary artery disease; FFR: fractional flow reserve

\section{References}

1. Tonino PA, De Bruyne B, Pijls NH, Siebert U, Ikeno F, van' t Veer M, Klauss V, et al. Fractional flow reserve versus angiography for guiding percutaneous coronary intervention. N Engl J Med. 2009;360(3):213-224.

2. Yang K, Yu SQ, Lu MJ, Zhao SH. Comparison of diagnostic accuracy of stress myocardial perfusion imaging for detecting hemodynamically significant coronary artery disease between cardiac magnetic resonance and nuclear medical imaging: A meta-analysis. Int J Cardiol. 2019;293:278-285.

3. Schuchlenz HW, Kuebeck M, Weihs W, et al. Myocardial perfusion scintigraphy or stress cardiac magnetic resonance: which is the ideal gatekeeper for the cathlab? An invasive evaluation in patients with stable coronary heart disease. Eur Heart J. 2010;31(suppl 1):584.

4. Bernhardt P, Walcher T, Rottbauer W, Wohrle J. Quantification of myocardial perfusion reserve at 1.5 and 3.0 Tesla: a comparison to fractional flow reserve. Int J Cardiovasc Imaging. 2012;28(8):2049-2056.

5. Bettencourt N, Chiribiri A, Schuster A, Ferreira N, Sampaio F, Duarte R, Santos L, et al. Cardiac magnetic resonance myocardial perfusion imaging for detection of functionally significant obstructive coronary artery disease: a prospective study. Int J Cardiol. 2013;168(2):765-773.

6. Chiribiri A, Hautvast GL, Lockie T, Schuster A, Bigalke B, Olivotti L, Redwood SR, et al. Assessment of coronary artery stenosis severity and location: quantitative analysis of transmural perfusion gradients by high-resolution MRI versus FFR. JACC Cardiovasc Imaging. 2013;6(5):600609.

7. Costa MA, Shoemaker S, Futamatsu H, Klassen C, Angiolillo DJ, Nguyen M, Siuciak A, et al. Quantitative magnetic resonance perfusion imaging detects anatomic and physiologic coronary artery disease as measured by coronary angiography and fractional flow reserve. J Am Coll Cardiol. 2007;50(6):514-522.

8. Ebersberger U, Makowski MR, Schoepf UJ, Platz U, Schmidtler F, Rose J, Kessel A, et al. Magnetic resonance myocardial perfusion imaging at 3.0 Tesla for the identification of myocardial ischaemia: comparison with coronary catheter angiography and fractional flow reserve measurements. Eur Heart J Cardiovasc Imaging. 2013;14(12):1174-1180.

9. Futamatsu H, Wilke N, Klassen C, Shoemaker S, Angiolillo DJ, Siuciak A, Morikawa-Futamatsu K, et al. Evaluation of cardiac magnetic resonance imaging parameters to detect anatomically and hemodynamically significant coronary artery disease. Am Heart J. 2007;154(2):298- 
305.

10. Ghekiere O, Dacher JN, Dewilde W, et al. Value of relative myocardial perfusion at MRI for fractional flow reserve - defined ischemia: a pilot study. American Journal of Roentgenology. 2019;212(5):1002-1009.

11. Groothuis JG, Beek AM, Brinckman SL, Meijerink MR, van den Oever ML, Hofman MB, van Kuijk C, et al. Combined non-invasive functional and anatomical diagnostic work-up in clinical practice: the magnetic resonance and computed tomography in suspected coronary artery disease (MARCC) study. Eur Heart J. 2013;34(26):19901998.

12. Hamada S, Gotschy A, Wissmann L, Paetsch I, Jahnke C, Plein S, Gebker R, et al. Multi-centre study of wholeheart dynamic 3D cardiac magnetic resonance perfusion imaging for the detection of coronary artery disease defined by fractional flow reserve: gender based analysis of diagnostic performance. Eur Heart J Cardiovasc Imaging. 2017;18(10):1099-1106.

13. Huber A, Sourbron S, Klauss V, Schaefer J, Bauner KU, Schweyer M, Reiser M, et al. Magnetic resonance perfusion of the myocardium: semiquantitative and quantitative evaluation in comparison with coronary angiography and fractional flow reserve. Invest Radiol. 2012;47(6):332338.

14. Jogiya R, Kozerke S, Morton G, De Silva K, Redwood S, Perera D, Nagel E, et al. Validation of dynamic 3-dimensional whole heart magnetic resonance myocardial perfusion imaging against fractional flow reserve for the detection of significant coronary artery disease. J Am Coll Cardiol. 2012;60(8):756-765.

15. Kamiya K, Sakakibara M, Asakawa N, Yamada S, Yoshitani $\mathrm{T}$, Iwano $\mathrm{H}$, Komatsu $\mathrm{H}$, et al. Cardiac magnetic resonance performs better in the detection of functionally significant coronary artery stenosis compared to singlephoton emission computed tomography and dobutamine stress echocardiography. Circ J. 2014;78(10):2468-2476.

16. Kirschbaum SW, Springeling T, Rossi A, Duckers E, Gutierrez-Chico JL, Regar E, de Feyter PJ, et al. Comparison of adenosine magnetic resonance perfusion imaging with invasive coronary flow reserve and fractional flow reserve in patients with suspected coronary artery disease. Int J Cardiol. 2011;147(1):184-186.

17. Kuhl HP, Katoh M, Buhr C, Krombach GA, Hoffmann R, Rassaf T, Neizel M, et al. Comparison of magnetic resonance perfusion imaging versus invasive fractional flow reserve for assessment of the hemodynamic significance of epicardial coronary artery stenosis. Am J Cardiol. 2007;99(8):1090-1095.

18. Lockie T, Ishida M, Perera D, Chiribiri A, De Silva K, Kozerke S, Marber M, et al. High-resolution magnetic resonance myocardial perfusion imaging at 3.0-Tesla to detect hemodynamically significant coronary stenoses as determined by fractional flow reserve. J Am Coll Cardiol. 2011;57(1):70-75.

19. Luu JM, Friedrich MG, Harker J, Dwyer N, Guensch D, Mikami Y, Faris P, et al. Relationship of vasodilatorinduced changes in myocardial oxygenation with the severity of coronary artery stenosis: a study using oxygen- ation-sensitive cardiovascular magnetic resonance. Eur Heart J Cardiovasc Imaging. 2014;15(12):1358-1367.

20. Manka R, Paetsch I, Kozerke S, Moccetti M, Hoffmann $\mathrm{R}$, Schroeder J, Reith S, et al. Whole-heart dynamic threedimensional magnetic resonance perfusion imaging for the detection of coronary artery disease defined by fractional flow reserve: determination of volumetric myocardial ischaemic burden and coronary lesion location. Eur Heart J. 2012;33(16):2016-2024.

21. Manka R, Wissmann L, Gebker R, Jogiya R, Motwani M, Frick M, Reinartz S, et al. Multicenter evaluation of dynamic three-dimensional magnetic resonance myocardial perfusion imaging for the detection of coronary artery disease defined by fractional flow reserve. Circ Cardiovasc Imaging. 2015;8(5):e003061.

22. Nakamori S, Sakuma H, Dohi K, Ishida M, Tanigawa T, Yamada A, Takase S, et al. Combined assessment of stress myocardial perfusion cardiovascular magnetic resonance and flow measurement in the coronary sinus improves prediction of functionally significant coronary stenosis determined by fractional flow reserve in multivessel disease. J Am Heart Assoc. 2018;7(3):e007736.

23. Nissen L, Winther S, Westra J, Ejlersen JA, Isaksen C, Rossi A, Holm NR, et al. Diagnosing coronary artery disease after a positive coronary computed tomography angiography: the Dan-NICAD open label, parallel, head to head, randomized controlled diagnostic accuracy trial of cardiovascular magnetic resonance and myocardial perfusion scintigraphy. Eur Heart J Cardiovasc Imaging. 2018;19(4):369-377.

24. Pan J, Huang S, Lu Z, Li J, Wan Q, Zhang J, Gao C, et al. Comparison of myocardial transmural perfusion gradient by magnetic resonance imaging to fractional flow reserve in patients with suspected coronary artery disease. Am J Cardiol. 2015;115(10):1333-1340.

25. Papanastasiou G, Williams MC, Dweck MR, Alam S, Cooper A, Mirsadraee S, Newby DE, et al. Quantitative assessment of myocardial blood flow in coronary artery disease by cardiovascular magnetic resonance: comparison of Fermi and distributed parameter modeling against invasive methods. J Cardiovasc Magn Reson. 2016;18(1):57.

26. Pereira E, Bettencourt N, Ferreira N, Schuster A, Chiribiri A, Primo J, Teixeira M, et al. Incremental value of adenosine stress cardiac magnetic resonance in coronary artery disease detection. Int J Cardiol. 2013;168(4):41604167.

27. Ponte M, Bettencourt N, Pereira E, Ferreira ND, Chiribiri A, Schuster A, Albuquerque A, et al. Anatomical versus functional assessment of coronary artery disease: direct comparison of computed tomography coronary angiography and magnetic resonance myocardial perfusion imaging in patients with intermediate pre-test probability. Int J Cardiovasc Imaging. 2014;30(8):1589-1597.

28. Rieber J, Huber A, Erhard I, Mueller S, Schweyer M, Koenig A, Schiele TM, et al. Cardiac magnetic resonance perfusion imaging for the functional assessment of coronary artery disease: a comparison with coronary angiography and fractional flow reserve. Eur Heart J. 
2006;27(12):1465-1471.

29. Walcher T, Manzke R, Hombach V, Rottbauer W, Wohrle J, Bernhardt P. Myocardial perfusion reserve assessed by T2-prepared steady-state free precession blood oxygen level-dependent magnetic resonance imaging in comparison to fractional flow reserve. Circ Cardiovasc Imaging. 2012;5(5):580-586.

30. Watkins S, McGeoch R, Lyne J, Steedman T, Good R, McLaughlin MJ, Cunningham T, et al. Validation of magnetic resonance myocardial perfusion imaging with fractional flow reserve for the detection of significant coronary heart disease. Circulation. 2009;120(22):2207-2213.

31. Pontone G, Andreini D, Baggiano A, Bertella E, Mushtaq $\mathrm{S}$, Conte E, Beltrama V, et al. Functional relevance of coronary artery disease by cardiac magnetic resonance and cardiac computed tomography: myocardial perfusion and fractional flow reserve. Biomed Res Int. 2015;2015:297696.

32. Plein S, Motwani M. Fractional flow reserve as the reference standard for myocardial perfusion studies: fool's gold? Eur Heart J Cardiovasc Imaging. 2013;14(12):12111213.

33. Desai RR, Jha S. Diagnostic performance of cardiac stress perfusion MRI in the detection of coronary ar- tery disease using fractional flow reserve as the reference standard: a meta-analysis. AJR Am J Roentgenol. 2013;201(2):W245-252.

34. Li M, Zhou T, Yang LF, Peng ZH, Ding J, Sun G. Diagnostic accuracy of myocardial magnetic resonance perfusion to diagnose ischemic stenosis with fractional flow reserve as reference: systematic review and meta-analysis. JACC Cardiovasc Imaging. 2014;7(11):1098-1105.

35. Jiang B, Cai W, Lv X, Liu H. Diagnostic performance and clinical utility of myocardial perfusion MRI for coronary artery disease with fractional flow reserve as the standard reference: a meta-analysis. Heart Lung Circ. 2016;25(10):1031-1038.

36. Danad I, Szymonifka J, Twisk JWR, Norgaard BL, Zarins CK, Knaapen P, Min JK. Diagnostic performance of cardiac imaging methods to diagnose ischaemia-causing coronary artery disease when directly compared with fractional flow reserve as a reference standard: a metaanalysis. Eur Heart J. 2017;38(13):991-998.

37. Dai N, Zhang X, Zhang Y, Hou L, Li W, Fan B, Zhang T, et al. Enhanced diagnostic utility achieved by myocardial blood analysis: A meta-analysis of noninvasive cardiac imaging in the detection of functional coronary artery disease. Int J Cardiol. 2016;221:665-673. 